\title{
Improving Dysarthria Classification by Pattern Recognition Techniques Based on a Bionic Model
}

\author{
Eduardo Gonzalez-Moreira, Diana Torres, Carlos A. Ferrer, and Yusely Ruiz \\ Center for Studies on Electronics and Information Technologies, \\ Central University of Las Villas, Cuba \\ \{moreira, dtb, cferrer, yuselyr\}@uclv.edu.cu
}

\begin{abstract}
The goal of this research is to use a bionic model to enhance classification of Dysarthria. The model based on the main features of the mammalian olfactory system is the initial stage of the recognition process. The bionic model aimed to achieve an enhancement in the separation ability of the dysarthric features. The recognition performance obtained by four different pattern recognition algorithms using the bionic model to improve the features is shown and discussed. The results indicated that bionic model had clear influence on classification performance of well-known techniques using dysarthria database as case study. We regard the results of this study as a promising initial step to the use of bionic model as a recognition improvement function.
\end{abstract}

Keywords: pattern recognition, bionic model, dysarthria.

\section{Introduction}

Nowadays one of the persistent problems in the study of pattern recognition is the efficient description of relevant features and the best selection of the artificial intelligent network to the classification tasks. Neural network theory is an old research topic that has been widely used in recent years. Computational power developments and mathematics of complexity have made the field succeed during the last years, and made a significant approach to simulate complex biological systems.

As one of the most important sensory modalities in the sensory systems of mammals, olfactory nervous systems have attracted many researchers during the last years. Some models have been developed to emulate the functions of olfactory nervous systems [1, 2]. The olfactory nervous system is relatively simple and well-known functionally and morphologically, and is an interesting system for understanding the cognitive processes performed in the brain. Even when some brain processes remains unclear, many aspects of olfaction, such as the mechanisms of reception and central processing or the nature of the stimuli have been fairly extensively studied [3, 4].

In helping to understand the olfactory information processing many mathematical models that mimic the main feature of the olfactory system have been applied to pattern recognition, often with remarkable results [5-7]. Therefore this is the approach followed in this research, where a novel methodology for dysarthria classification is designed and implemented. 
Dysarthria is a term associated with a group of neurological diseases caused by lesions in the peripheral or central nervous system. Different speech perturbations are associated to the type and location of the lesions, which are correlated with both: the kind of dysarthria and the brain damage. The speech is one of the mechanisms that are more sensitive to lesions in the nervous system due to the precise coordination and timing required for normal speech production. Therefore, the study of the speech in patients suffering this pathology can reveal important information for assessment and treatment, increasing the reliability and effectiveness of the diagnosis process.

The goal of the present research is to introduce a bionic model, inspired on the mammalian olfactory system, to improve the classification process through enhancement of the data under analysis. The bionic model is formed from a bulbar model which mimics the behavior of excitatory mitral and inhibitory granule cells, and a 3layered cortical model, which emulates the structure and behavior of the piriform cortex $[8,9]$. Unsupervised bionic model allows for the weightings of the input acoustic measures to be determined on the basis of the inherent nonlinear regularities of the input data space. Unsupervised learning is particularly advantageous when no gold standard exists for classification. It is hoped that by using a bionic model, which is trained using few samples, will provide an upgrading to the data resulting in a better classification result.

\section{Bionic Model}

The bionic model emulates the main structural features of the olfactory system mimicking two main parts, bulb and cortex, and how they are connected via feedforward and feedback channels (Fig. 1). The researchers look for a balance between the wish for realism when comparing theoretical and computational results with experiment, and the need for abstraction and simplification of the biological complexity for a mathematical analysis and computer simulation.

The olfactory bulb can be viewed as the first central olfactory relay station extracting specific stimulus features, a function characteristic of the primary sensory areas in the brain $[10,11]$. The cellular structure of the bulb is well established and in this work, the olfactory bulb was modeled using a simple approximation of excitatory mitral and inhibitory granule cells. The activity of mitral cells was spatially distributed such that odorants were represented in the bulb model by a distributed pattern of mitral cell activity $[12,13]$. Mitral cells adjacent to each other project to the same or neighboring glomerulus. Among these models, the dynamics of every neural ensemble is described using a second order differential equation (Eq. (1)), based on physiological experiments of the olfactory system [14]:

$$
\frac{1}{a b}\left[x_{i}^{\prime \prime}(t)+(a+b) x_{i}^{\prime}(t)+a b x_{i}(t)\right]=\sum_{j \neq i}^{N}\left[W_{i j} q\left(x_{j}(t), g_{j}\right)\right]+I_{i}(t)+\varepsilon(t)
$$

Here $i=1, \ldots, N$, where $N$ is the number of channels, $x_{i}(t)$ indicates the state variable of $i$ th neural ensemble, $x_{j}(t)$ represent the state variable of $j$ th neural ensemble, which is connected to the $i$ th, $W_{i j}$ represents the connection strength between them. $I_{i}(t)$ is an input function which stands for the external input to the $i$ th channel. $\varepsilon(t)$ is 
noise or spontaneous neural activity. The parameters $a$ and $b$ reflect two rate constants. $q\left(x_{j}(t), g_{j}\right)$ is a static nonlinear sigmoid function derived from the Freeman model [1] and $g$ represents the maximum asymptote of the sigmoid function, also experimentally obtained from biological trials. However, the exact form of these relations is not essential to the system behavior, as long as the shape is qualitatively conserved. Since granule cells do not have axons, they are modeled using a larger linear range, and thus a less strong nonlinear threshold effect than for mitral cells.

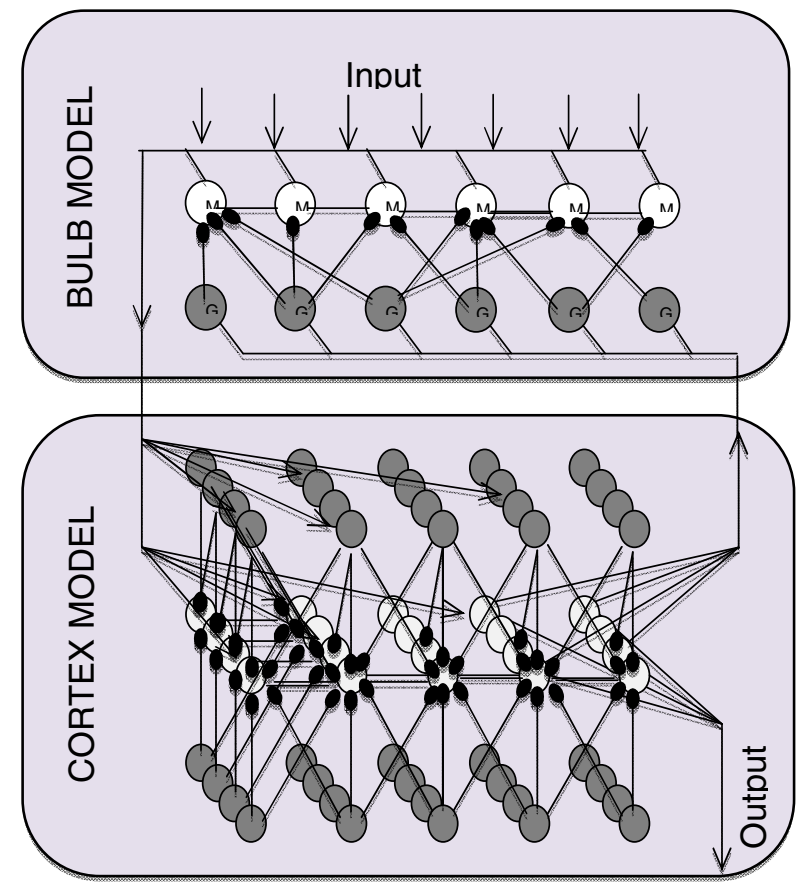

Fig. 1. Bionic model structure consists of two main parts (bulb model and cortex model) and their connections

Basically, the piriform cortex structures belonging to the allocortex are thinner and structurally less complex (having three cortical layers) than the neocortex $[15,16]$. The architecture of the cortical model was based on the 3-layered structure of the olfactory piriform cortex, using similar network connectivity, but relatively simple model nodes, representing populations of neurons. The two sets of inhibitory nodes have two different time constants and slightly different connectivity to the excitatory nodes. All connections were modeled with distance dependent time delays for signal propagation, corresponding to the geometry and fiber characteristics of the real cortex.

Similar to previously described for bulb model, the time evolution for a cortical network of $N$ neural ensemble is given by a set of coupled nonlinear first order differential equations for all internal states, $u$. With external input, coming from the bulb, $I(t)$, noise or spontaneous neural activity $\varepsilon(t)$, characteristic time constant $c$, 
and connection weights $W_{i j}$ between units $i$ and $j$, separated with a time delay $\delta_{i j}$, the neural activity for each ensemble, $u_{i}$, is given by:

$$
\frac{1}{c}\left[c u_{i}^{\prime}(t)+u_{i}(t)\right]=\sum_{j \neq i}^{N}\left[W_{i j} q_{j}\left(u_{j}\left(t-\delta_{i j}\right)\right)\right]+I_{i}(t)+\varepsilon(t)
$$

The continuous sigmoid function, $q_{j}\left(u_{j}\right)$, represents the input-output transfer function, experimentally determined by Freeman [1]. With gain parameter $g_{j}$, determines the slope, threshold, and amplitude of the curve for $j$ th ensemble and $D$ a normalization constant, $q_{j}$, is described by:

$$
\left.q_{j}=D g_{j}\left\{1-\exp \left[\frac{-\exp \left(u_{j}\right)-1}{g_{j}}\right]\right\}\right)
$$

It has been previously shown that the model displays major characteristics of the olfactory cortex dynamics. A typical EEG (encephalogram) time series from the cat olfactory cortex is shown in Fig. 2, together with a simulated EEG trace using the current model. Oscillatory and aperiodic dynamic behavior was shown to improve the performance by reducing the recall (convergence) time in associative memory tasks.
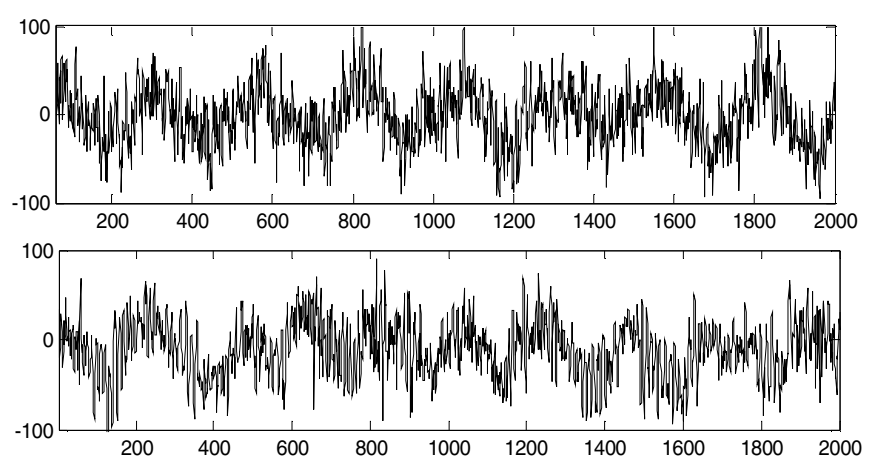

Fig. 2. Simulated (top) and real (bottom) EEG, showing the complex dynamics of cortical structures. The real trace is from cat olfactory bulb (data courtesy of Walter J. Freeman), whereas the simulated trace is from a simulation with the current cortical model. The $\mathrm{x}$-axis shows milliseconds, and the y-axis is in microvolts.

While time constants, signal velocities, and other system parameters are determined by physiological constraints, the connection weights should be adjusted properly for the best performance of the model. The bionic model presents two learning processes: Hebbian associative learning and habituation. These learning processes exist in a subtle balance and their relative importance changes at various stages of the memory process. The memory basins and attractors are formed via Hebbian learning under reinforcement, while the impact of environment noise, which includes the background inputs without any information, is reduced by habituation.

The learning processes are applied to the bulb model and to the cortex model. According to modified Hebbian rule, each pair of nodes co-activated by the stimulus 
have their lateral connections strengthened. The nodes with activities larger than the mean on the layer are considered as activated ones and strengthened with the Hebbian coefficient. In contrast, those with activity less than the mean are not considered to be activated ones and these connections are decreased by the Habituation coefficient and the simulation period. A bias coefficient is defined in the modified Hebbian learning rule to avoid the weight space saturation. These processes are applied in bulbar mitral layer and middle cortical layer.

\section{Case Study}

This study was carried out using speech datasets that contain records from eight types of dysarthria among which are: Spastic Dysarthria, Flaccid Dysarthria, Ataxic Dysarthria, Hyperkinetic Dysarthria (organic voice tremor, chorea and dystonia), Hypokinetic Dysarthria (Parkinson disease) and Mixed Dysarthria (Amyotrophic Lateral Sclerosis). For the particular case study analyzed in this research each kind of dysarthria defines one class in the classification task and comprehend an average of 14 subjects taken from 2 databases corresponding with different levels of the severity of the illnesses. The kind of severity of the sickness is annotated in the databases, where a total of 38, both perceptual and acoustic features, are also given. The first dataset was created including 62 patients [17]. The second dataset was a selection of dysarthric speakers from a database collected by Aronson and colleagues [18]. A total of 14 normal subjects were used as control to contrast the differences between the pathologic and normal speech.

The perceptual and acoustical measures contained in both databases were obtained from 3 utterances that provide more information about these diseases with less computational and storage requirements [19]. The utterances consisted of the sustained phonation of the vowel /a/, the repetition of the syllable /PA/TA/KA/ and the reading of the passage: 'The Grandfather' [20].

Therefore a total of 127 samples were analyzed to classify subjects into nine distinct groups, eight dysarthric groups and a control group. From a total of 38 features, 36 (25 perceptual and 11 acoustic) were selected to provide information about the condition of the speech mechanism of dysarthric patients [21]. However, a multidimensional analysis of dysarthric speech revealed that not all of the 36 features provided valuable information about the dysarthric groups [17].

From this point of view, some linear analysis techniques (clustering of variables, linear discriminant analysis, best first) were applied in order to obtain a reduction in the number of features of the data $[17,22]$. The resultant number of features was set to 20,16 and 12 after dimensionality reduction, following a commonly used rule in pattern recognition that states a ratio of at least 10 cases per input observation. Finally four versions of the original databases were created with $36,20,16$ and 12 features respectively.

\section{$4 \quad$ Results}

In this paper, we preprocess the four databases with the bionic model prior to the classification stage (Fig. 3). The bionic model transforms input features according to 
its nonlinear dynamics. This means that original features entering the mitral layer are modified inside the bionic model, and delivered by the middle cortical layer to the next stage.

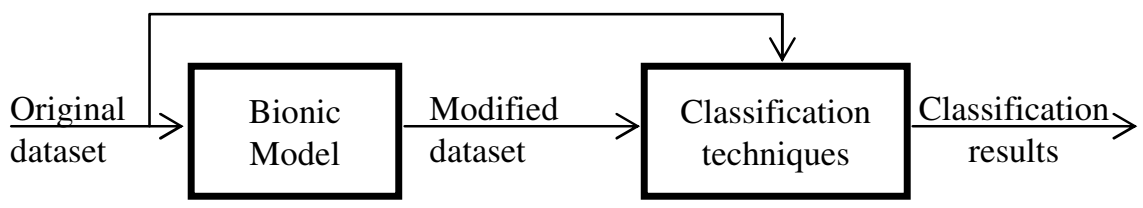

Fig. 3. Experimental setup for evaluating the influence of the bionic model performance in the final classification results using dysarthria databases

In the final classification stage four well-known techniques like Support Vector Machines (SVM), Bayesian Network (BN), decision trees (J48) and Naive Bayes (NB) were applied. These machine learning algorithms are included into the data mining software WEKA [23]. The databases are randomly reordered and then split into $n$ folds of equal size. The leave-one-out cross-validation method is applied. In our case $n$ is equal to the number of examples $(n=127)$, and in each iteration of the crossvalidation method, one fold is used for testing and the other $n-1$ folds is used for training the classifier.

Table 1. Percent of correct clasification of various methods with original and modified datasets, along with their corresponding MSSS as an improvement index

\begin{tabular}{lcccc}
\hline Methods & \multicolumn{4}{c}{ Dataset dimensions } \\
\cline { 2 - 5 } SVM + original data & $\mathbf{1 2}$ & 16 & 20 & 36 \\
SVM + modified data & 79.50 & 79.10 & 81.20 & 80.30 \\
MSSS (\%) & 81.10 & 79.50 & 79.50 & 82.80 \\
J48 + original data & $\mathbf{7 . 8 0}$ & $\mathbf{1 . 9 1}$ & $\mathbf{- 9 . 0 4}$ & $\mathbf{1 2 . 6 9}$ \\
J48 + modified data & 66.10 & 66.00 & 68.50 & 62.20 \\
MSSS (\%) & 72.40 & 72.50 & 70.10 & 68.50 \\
BN + original data & $\mathbf{1 8 . 5 8}$ & $\mathbf{1 9 . 1 2}$ & $\mathbf{5 . 0 8}$ & $\mathbf{1 6 . 6 7}$ \\
BN + modified data & 80.20 & 81.10 & 81.80 & 80.10 \\
MSSS (\%) & 83.50 & 85.50 & 81.10 & 83.30 \\
NB + original data & $\mathbf{1 6 . 6 7}$ & $\mathbf{2 3 . 2 8}$ & $\mathbf{- 3 . 8 5}$ & $\mathbf{1 6 . 0 8}$ \\
NB + modified data & 70.00 & 71.60 & 66.10 & 64.90 \\
MSSS (\%) & 73.20 & 72.90 & 68.50 & 65.40 \\
\hline
\end{tabular}

The four datasets containing objective and perceptual judgments were evaluated with respect to the percentage of correct classification provided by each classifier using bionic model to pre-process the data and without using it. Moreover a skill score based on mean squared error (MSSS) is used as an improvement index in order to evaluate the impact of the bionic model in the final classification results. MSSS is defined as one minus the ratio of the squared error for the classification with modified 
datasets to the squared error for the classification with original datasets. The result of the assessment process revealed that the bionic model allowed an improved classification rate over the original databases in almost every trial, as shown in Table 1.

These results show that the bionic model implementation is appropriate to enhance the classification of the dysarthric groups studied, providing a better percentage of correct classification in almost every trial. In only two cases the bionic models worsened classification results. Particularly two of the four algorithms take significant advantage from the modified features introduced by the bionic model in the initial state of the data processing, J48 and BN. In general, modified dataset based on the bionic model outperforms the performance of almost every classification techniques.

\section{Conclusions}

In this work, a bionic model mimicking the main features of the olfactory system has been analyzed, and its performance to improve dysarthric classification was shown. Our bionic model is constructed from two principal parts: a bulb model and a cortex model. The bulb model is composed of mitral and granule cells, whereas the cortical model mimics the 3-layered structure of the mammalian piriform cortex.

The analyses performed have revealed that the model has the capacity to learn complex patterns due to Hebbian modification of the connection strengths of the Msets excitatory synapses in the bulb and the excitatory units on the cortical middle layer. The improvement in the classification of the dysarthric databases was shown.

The current digital computers are the bottleneck for the models based on biological sensorial systems due to the time required to solve ODE by numerical integration. Nevertheless, even when considering the memory requirements and the computational time demanded, the bionic model still cannot compete against conventional classification methods. The results obtained with this research have become a promising initial step into the use of the bionic model to improve other patter recognition tasks.

Acknowledgment. The authors would like to thank Julian Cardenas Barrera, Guang Li, Hans Liljenström and Walter J. Freeman for their support to this research.

\section{References}

1. Freeman, W.J.: Mass action in the nervous system. Academic Press, New York (1975)

2. Shepherd, G., Brayton, R.: Computer simulation of a dendrodendritic synaptic circuit for self- and lateral-inhibition in the olfactory bulb. Brain Res. 175, 377-382 (1979)

3. Freeman, W.J., Skarda, C.: Spatial EEG patterns, non-linear dynamics and perception: the neo-sherringtonian view. Brain Res. Rev. 10, 147-175 (1985)

4. Aronsson, P., Liljenstrom, H.: Effects of non-synaptic neuronal interaction in cortex on synchronization and learning. Biosystems 63, 43-56 (2001) 
5. Liljenström, H.: Stability and instability in autonomous systems. In: Advances in Cognitive Neurodynamics - Proceedings of the International Conference on Cognitive Neurodynamics, Shanghai, November 17-21, pp. 661-665 (2008)

6. Kozma, R., Freeman, W.J.: The KIV Model of Intentional Dynamics and Decision Making. Neural Networks 22(3), 277-285 (2009)

7. Gonzalez-Moreira, E., Li, G., Ruiz, Y.: A tea classification method based on an olfactory system model. In: Advances in Cognitive Neurodynamics, Hangzhou, pp. 747-751 (2008)

8. Gonzalez-Moreira, E., Liljenstrom, H., Ruiz, Y., Li, G.: A biological inspired model for pattern recognition. J. Zhejiang Univ. Sci. B 11, 115-126 (2010)

9. Gonzalez-Moreira, E., Ruiz, Y.: A bionic model inspired on the olfactory system. In: V Latin American Congress on Biomedical Engineering (CLAIB 2011), Havana (2011)

10. Doty, R.: Handbook of olfaction and gustation. Dekker, New York (2003)

11. Lowe, G.: Electrical signaling in the olfactory bulb. Curr. Opin. Neurobiol. 13, 476-481 (2003)

12. Mori, K., Nagao, H., Yoshihara, Y.: The olfactory bulb: coding and processing of odor molecule information. Science 286, 711-715 (1999)

13. Leon, M., Johnson, B.: Olfactory coding in the mammalian olfactory bulb. Brain Res. Rev. 42, 23-32 (2003)

14. Freeman, W.J.: Nonlinear gain mediating cortical stimulus-response relations. Biol. Cybernet. 33, 237-247 (1979)

15. Liljenstrom, H.: Modeling the dynamics of olfactory cortex using simplified network units and realistic architecture. Int. J. Neural Syst. 2, 1-15 (1991)

16. Kowianski, P., Lipowska, M., Morys, J.: The piriform cortex and the endopiriform nucleus in the rat reveal generally similar pattern of connections. Folia Morphol. 58, 9-19 (1999)

17. Castillo Guerra, E.: A modern approach to dysarthria classification. Ph.D. Thesis at UNB, Canada (2002)

18. Aronson, A.E.: Dysarthrias: differential diagnosis (1993)

19. Darley, F.L., Aronson, A.E., Brown, J.R.: Differential diagnostic patterns of dysarthria. J. Speech Hear Res. 12, 246-249 (1969)

20. Darley, F.L., Aronson, A.E., Brown, J.R.: Motor speech disorders, 3rd edn. W.B. Saunders Company, Philadelphia (1975)

21. Castillo Guerra, E., Lovey, D.F.: A modern approach to dysarthria classification. In: 25th Annual lntemational Conference of the IEEE EMBS, Cancun (2003)

22. Torres, D., Acebo, L., Gonzalez-Moreira, E., Ferrer, C.A.: Uso de la señal de voz en la clasificación de disartrias. Presented at the RECPAT 2012 (2012)

23. Hall, M., Frank, E., Holmes, G., Pfahringer, B., Reutemann, P., Witten, I.H.: The WEKA data mining software: An update. Sigkdd Explor. 11 (2009) 